\title{
UPPER GI SURGERY
}

\section{Routes of early enteral nutrition following oesophagectomy}

\author{
M Elshaer ${ }^{1}$, G Gravante ${ }^{2}$, J White ${ }^{1}$, J Livingstone ${ }^{1}$, A Riaz ${ }^{1}$, A Al-Bahrani ${ }^{1}$ \\ ${ }^{1}$ West Hertfordshire Hospitals NHS Trust, UK \\ ${ }^{2}$ University Hospitals of Leicester NHS Trust, UK
}

\section{ABSTRACT}

INTRODUCTION Oesophagectomy for cancer is a challenging procedure with a five-year overall survival rate of 15-20\%. Early enteral nutrition following oesophagectomy is a crucial component of the postoperative recovery and carries a significant impact on the outcome. Different methods of enteral feeding were conducted in our unit. The aim of this study was to examine the efficacy and safety of nasojejunal tube (NJT), jejunostomy tube (JT) and pharyngostomy tube (PT) feeding after oesophagectomy.

METHODS A retrospective review was carried out of prospectively collected data on patients with oesophageal cancer who underwent an oesophagectomy between 2011 and 2014. The primary outcome was feeding tube related complications such as occlusion, dislocation and leak. The secondary outcomes were length of stay and 30-day morbidity.

RESULTS A total of 90 oesophagectomies were included in the study. A NJT was inserted in 41 patients (45.6\%), a JT was used in 14 patients (15.5\%) and a PT was the route for enteral nutrition in 35 patients (38.9\%). In total, five patients (5.5\%) developed tube related complications. There were no tube related complications in the NJT group but one JT patient (7.1\%) developed tube related cellulitis $(p=0.189)$ and four PT patients $(11.4 \%)$ developed tube related haemorrhage $(p=0.544)$, tube dislocation ( $p=0.544$ ) or cellulitis ( $p=0.189$ ). The median length of stay and 30-day postoperative morbidity were similar between the groups. CONCLUSIONS NJT feeding is a less invasive, feasible route for early enteral nutrition following oesophagectomy. A randomised controlled trial is recommended to verify these findings.

\section{KEYWORDS}

Oesophagectomy - Nasojejunal tube - Jejunostomy tube - Pharyngostomy tube

Accepted 16 April 2016

CORRESPONDENCE TO

Mohamed Elshaer, E: mohamedelshaer_1@hotmail.com

Approximately 400,000 people die from oesophageal cancer worldwide every year, equating to $5 \%$ of all cancer related mortality. ${ }^{1}$ The clinical presentation is often late and diagnosis is made at an advanced stage.

The aim of surgery in oesophageal cancer is to achieve curative resection and to accomplish a functioning anastomosis. Several factors affect the outcome following surgery such as respiratory complications, anastomotic leak and the postoperative nutritional status of the patient, which has a direct impact on the healing process and recovery. ${ }^{2}$ Patients with oesophageal cancer are likely to suffer from weight loss and malnutrition secondary to an obstructive tumour. Following oesophageal resection, these patients will remain without oral feeding for 5-7 days. Early enteral nutrition (EN) was therefore advocated to improve nutritional outcomes and decrease the incidence of postoperative complications. ${ }^{3}$ Early EN has been found to decrease the length of hospital stay (LOS) and lead to a better outcome than parenteral nutrition (PN), in addition to reducing the total cost of patient care. ${ }^{4,5}$

Early EN following oesophagectomy is usually maintained via a nasojejunal tube (NJT), a jejunostomy tube (JT) or a pharyngostomy tube (PT). The use of a NJT for EN after oesophagectomy was first reported by Page et al; however, this method was compared only with PN. ${ }^{6}$ Conversely, another trial compared NJT versus JT feeding following oesophagectomy. ${ }^{7}$ There was no evidence to suggest that one technique should be favoured over the other.

Although PT feeding is not a new technique, it has not been compared with NJT or JT feeding previously. The use of EN is not without complications, and feeding tube related obstruction, dislocation, cellulitis and return to theatre have been reported. ${ }^{7,8}$ Another drawback is prolonged ileus or patients' intolerance to EN, which has resulted in feeding cessation in some cases. ${ }^{4}$ Every technique has its own benefits and risks, and the choice of feeding method is often at the surgeon's discretion. The aim of this study was to evaluate the outcomes of three routes for EN following oesophagectomy in our centre.

\section{Methods}

Patients who underwent oesophagectomies at West Hertfordshire Hospitals NHS Trust between 2011 and 2014 were 
reviewed. The upper gastrointestinal (GI) unit has a prospectively maintained database that includes basic demographics and clinical data such as age, sex, date of surgery, tumour site, type of operation performed and tumour histology. All patients who underwent open or laparoscopic (hybrid) transthoracic (Ivor Lewis, McKeown) oesophagectomies for cancer and who had early EN during the study period were included. Patients were excluded if they underwent oesophageal surgery for benign disease or trauma, as were those who underwent palliative resections.

Three techniques were used in our institution. The triple lumen NJT (Freka ${ }^{\circledR}$ Trelumina, 150cm, 9/16Fr; Fresenius Kabi AG, Bad Homburg, Germany) consists of a feeding lumen for jejunal feeding, a decompression lumen for simultaneous decompression and a ventilation lumen for ventilation during active suction (Fig 1). The NJT is inserted at the end of the procedure, using an endoscope to place it in the proximal jejunum. The tube is secured to the patient's nose with a silk suture and adhesive tape. Feeding is started 24 hours after surgery using a standard protocol.

A needle catheter technique is employed for insertion of the JT (Freka ${ }^{\circledR}, 75 \mathrm{~cm}, 9 \mathrm{Fr}$; Fresenius Kabi; Fig 2). A 14G long split cannula/introducer (white, $13 \mathrm{~cm}$ ) is inserted intramurally into the wall of the proximal jejunum for approximately $5 \mathrm{~cm}$ and the tip of the cannula/introducer is then inserted into the lumen. The introducer is removed and the JT is threaded through the needle for $25-30 \mathrm{~cm}$. The needle is withdrawn, and the JT is secured with purse string and seromuscular sutures. Following this, a short split needle (blue, $10.5 \mathrm{~cm}$ ) is inserted at $45^{\circ}$ through the anterior abdominal wall and the other end of the JT is threaded backwards to exit

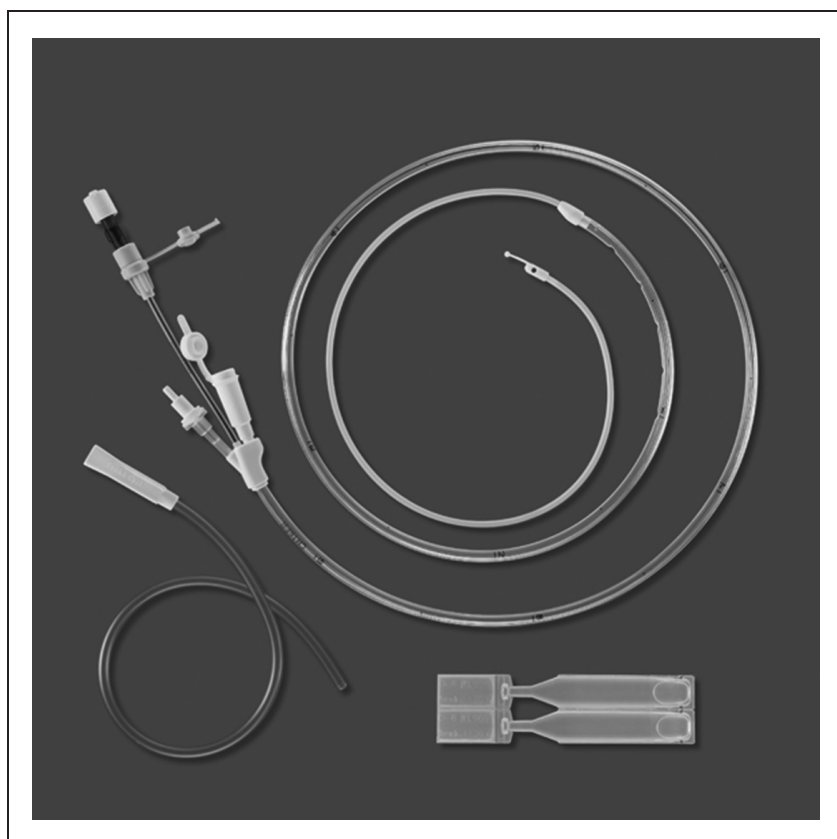

Figure 1 Freka ${ }^{\circledR}$ Trelumina nasojejunal tube (reproduced with permission from Fresenius Kabi)

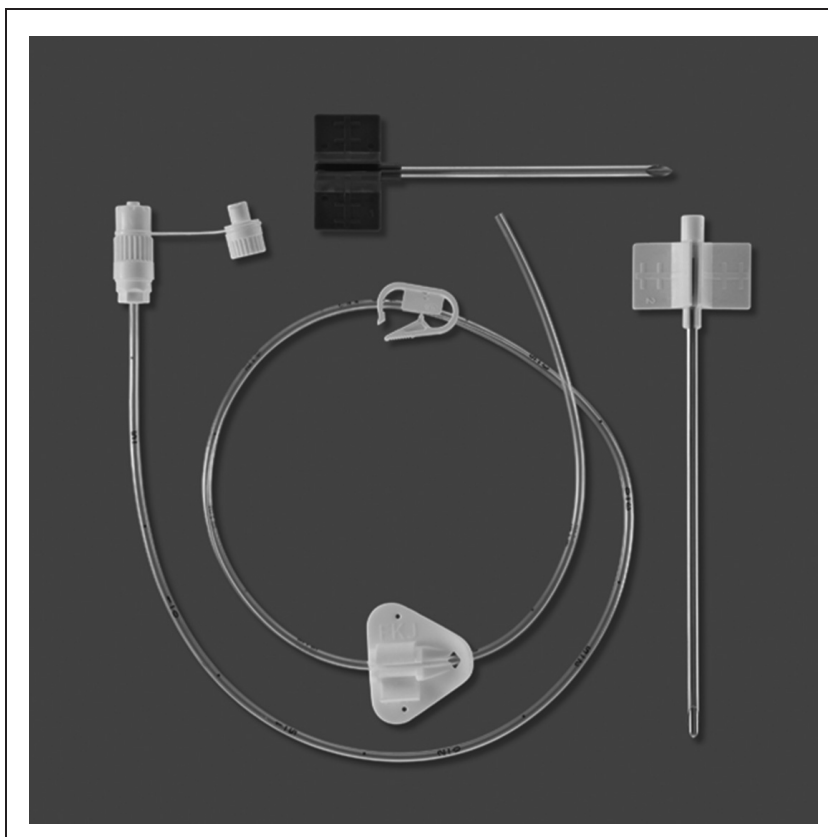

Figure 2 Freka ${ }^{\circledR}$ surgical jejunostomy set (reproduced with permission from Fresenius Kabi)

the abdomen. The needle is then split and removed, and the jejunum is sutured to the anterior abdominal wall. The JT is secured to the skin with a non-absorbable suture.

The triple lumen PT (Freka ${ }^{\circledR}$ Trelumina) is inserted using an artery forceps directed laterally into the pyriform recess, located behind the posterior tonsillar pillar, inferior and medial to the greater cornu of the hyoid bone. A small incision is made laterally in the neck over the tip of the artery forceps. The PT is grasped with a clamp and retracted from the mouth, and then grasped with an endoscopic forceps to be placed in the proximal jejunum. The tube is sutured to the skin of the neck (Fig 3).

EN was discontinued before discharge in all groups and all feeding tubes were removed prior to discharge except for the JT. JT patients were reviewed in the outpatient clinic after two weeks to evaluate their need for supplementary feeding. If prolonged feeding was anticipated, a JT was the preferred EN route.

Case notes and the hospital electronic database were used to collect data on patients who met the inclusion criteria. Data collected comprised ASA (American Society of Anesthesiologists) grade, body mass index, route of EN, anastomotic leak, respiratory complications, wound complications, feeding tube related complications, LOS and 30-day morbidity. The primary outcome was feeding tube related complications such as occlusion, dislocation and leak. The secondary outcomes were 30-day morbidity and LOS.

\section{Statistical analysis}

All data were inserted into Excel ${ }^{\circledR}$ (Microsoft, Redmond, WA, US) and analysed with SPSS ${ }^{\circledR}$ version 20.0 (IBM, New York, 


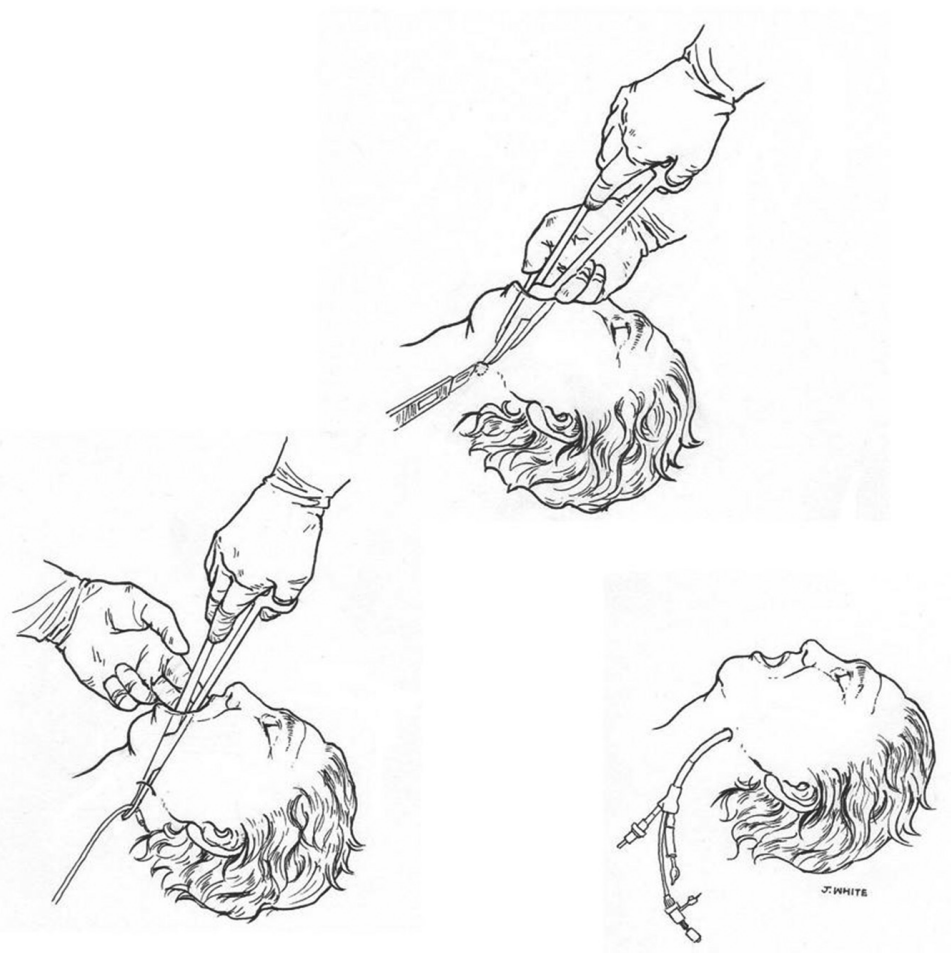

Figure 3 Pharyngostomy tube insertion technique

US). Normality assumptions were demonstrated with histograms and the Kolmogorov-Smirnov test. Differences between groups were examined with one-way analysis of variance for continuous parametric variables, the Kruskal-Wallis test for continuous non-parametric variables and the chisquared test for categorical variables (Fisher's exact test if cell count was $<5$ ). A $p$-value of $<0.05$ was considered statistically significant.

\section{Results}

A total of 90 oesophagectomies were performed during the study period. There were 73 men $(81.1 \%)$ and 17 women $(18.9 \%)$. The median age was 66 years (range: $41-80$ years). Nine patients $(10 \%)$ were current smokers, thirty-nine $(43 \%)$ were ex-smokers and forty-two $(47 \%)$ were nonsmokers. The body mass index was $19.0-27.5 \mathrm{~kg} / \mathrm{m}^{2}$ in 26 patients $(29 \%), 28.0-30.5 \mathrm{~kg} / \mathrm{m}^{2}$ in 39 patients $(43 \%)$ and $31.0-38.0 \mathrm{~kg} / \mathrm{m}^{2}$ in 25 patients $(28 \%)$.

All patients underwent preoperative oesophagogastroduodenoscopy. Staging with computed tomography (CT) was performed in all patients, positron emission tomography CT in 87 patients $(97 \%)$, endoscopic ultrasonography in 47 patients $(52 \%)$ and laparoscopy in 79 patients $(88 \%)$. The Eastern Cooperative Oncology Group performance status was 0 in 68 patients $(76 \%), 1$ in 15 patients $(17 \%)$, and 2 in seven $7(8 \%)$. In terms of ASA grade, 9 patients (10\%) were classified as grade 1, 57 patients $(63 \%)$ were grade 2,22 patients $(24 \%)$ were grade 3 and 2 patients ( $2 \%$ ) were grade 4 .

An Ivor Lewis two-stage oesophagectomy was performed in 88 patients $(98 \%)$ while a three-stage oesophagectomy was undertaken in 2 patients $(2 \%)$. The abdominal phase was performed laparoscopically in 46 patients (51\%) and using an open approach in 42 cases $(47 \%)$. A further two patients $(2 \%)$ had their procedure converted from laparoscopic to open. The thoracic phase was performed using a right thoracotomy in 89 cases and the thoracoscopic procedure of 1 patient was converted to an open procedure. Histological examination revealed the presence of an adenocarcinoma in 82 patients $(91.1 \%)$, a squamous carcinoma in 6 patients $(6.6 \%)$, a signet ring cell adenocarcinoma in 1 patient $(1.1 \%)$ and an undifferentiated basaloid carcinoma in 1 patient $(1.1 \%)$.

\section{Feeding tubes}

Patient characteristics and demographics are illustrated in Table 1. A NJT was inserted in 41 patients $(45.6 \%)$, a JT in 14 patients $(15.5 \%)$ and a PT in 35 patients $(38.9 \%)$. A statistically significant difference was present between the feeding route and the approach for the abdominal phase (laparoscopic or open). For most of the operations involving a NJT, the abdominal phase was conducted laparoscopically while for JT and PT patients, the abdominal phase was conducted more frequently using an open approach (Fisher's exact test, $p=0.043$ ) (Table 1). 
Table 1 Patient characteristics and demographics

\begin{tabular}{|c|c|c|c|c|}
\hline Variables & NJT $(n=41)$ & JT $(n=14)$ & PT $(n=35)$ & $p$-value \\
\hline Mean age (years) & 66.2 (SD: 10.1) & 63.4 (SD: 8.5) & 63.7 (SD: 10.1) & 0.469 \\
\hline $\begin{array}{l}\text { ASA grade } \\
1 \\
2 \\
3 \\
4\end{array}$ & $\begin{array}{l}8(19.5 \%) \\
25(61.0 \%) \\
8(19.5 \%) \\
0(0 \%)\end{array}$ & $\begin{array}{l}1(7.1 \%) \\
8(57.1 \%) \\
5(35.7 \%) \\
0(0 \%)\end{array}$ & $\begin{array}{l}0(0 \%) \\
24(68.6 \%) \\
9(25.7 \%) \\
2(5.7 \%)\end{array}$ & 0.043 \\
\hline $\begin{array}{l}\text { ECOG performance status } \\
0 \\
1 \\
2\end{array}$ & $\begin{array}{l}29(70.7 \%) \\
7(17.1 \%) \\
5(12.2 \%)\end{array}$ & $\begin{array}{l}8(57.1 \%) \\
5(35.7 \%) \\
1(7.1 \%)\end{array}$ & $\begin{array}{l}19(54.3 \%) \\
14(40.0 \%) \\
2(5.7 \%)\end{array}$ & 0.206 \\
\hline Mean BMI $\left(\mathrm{kg} / \mathrm{m}^{2}\right)$ & 26.5 (SD: 3.3) & 26.8 (SD: 3.8) & 28.3 (SD: 3.6) & 0.069 \\
\hline Neoadjuvant CRT & $20(48.8 \%)$ & $10(71.4 \%)$ & $18(51.4 \%)$ & 0.171 \\
\hline $\begin{array}{l}\text { Abdominal phase } \\
\text { Laparoscopic } \\
\text { Converted to open } \\
\text { Open }\end{array}$ & $\begin{array}{l}34(82.9 \%) \\
0(0 \%) \\
7(17.1 \%)\end{array}$ & $\begin{array}{l}2(14.3 \%) \\
1(7.1 \%) \\
11(78.6 \%)\end{array}$ & $\begin{array}{l}10(28.6 \%) \\
1(2.9 \%) \\
24(68.6 \%)\end{array}$ & 0.001 \\
\hline Adjuvant therapy & $14(34.1 \%)$ & $7(50.0 \%)$ & $16(45.7 \%)$ & 0.418 \\
\hline
\end{tabular}

Table 2 30-day morbidity by type of feeding tube

\begin{tabular}{|c|c|c|c|c|}
\hline Complications & NJT $(n=41)$ & $\mathrm{JT}(n=14)$ & PT $(n=35)$ & $p$-value \\
\hline Tube related cellulitis $(n=3)$ & $0(0 \%)$ & $1(7.1 \%)$ & $2(5.7 \%)$ & 0.189 \\
\hline Tube haemorrhage $(n=1)$ & $0(0 \%)$ & $0(0 \%)$ & $1(2.9 \%)$ & 0.544 \\
\hline Tube occlusion or dislocation $(n=1)$ & $0(0 \%)$ & $0(0 \%)$ & $1(2.9 \%)$ & 0.544 \\
\hline Total $(n=5)$ & $0(0 \%)$ & $1(7.1 \%)$ & $4(11.4 \%)$ & 0.063 \\
\hline Respiratory complications ( $n=20$ ) & $9(22.0 \%)$ & $4(28.6 \%)$ & $7(20.0 \%)$ & 0.792 \\
\hline Reoperation ( $n=13$ ) & $6(14.6 \%)$ & $2(14.3 \%)$ & $5(14.3 \%)$ & 1.000 \\
\hline Cardiac complications $(n=7)$ & $2(4.9 \%)$ & $2(14.3 \%)$ & $3(8.6 \%)$ & 0.435 \\
\hline Chyle leak $(n=5)$ & $3(7.3 \%)$ & $0(0 \%)$ & $2(5.7 \%)$ & 0.845 \\
\hline Anastomotic leak $(n=4)$ & $3(7.3 \%)$ & $1(7.1 \%)$ & $0(0 \%)$ & 0.267 \\
\hline Haemorrhage $(n=3)$ & $1(2.4 \%)$ & $0(0 \%)$ & $2(5.7 \%)$ & 0.756 \\
\hline Gastric conduit necrosis $(n=1)$ & $0(0 \%)$ & $0(0 \%)$ & $1(2.9 \%)$ & 0.544 \\
\hline Total $(n=53)$ & 24 (58.5\%) & 9 (64.3\%) & 20 (57.1\%) & 0.918 \\
\hline
\end{tabular}

\section{Complications}

Tube related complications and 30-day morbidity are reported in Table 2. Five patients (5.5\%) developed tube related complications. No statistically significant differences were present among the groups for 30-day morbidity but there was a trend towards significance for tube related complications with PT compared with NJT and JT feeding $(p=0.063)$.

\section{Length of stay}

The median LOS for the entire cohort was 11 days (range: 2-75 days). In terms of the individual groups, it was 12 days (range: 6-75 days) for NJT patients, 11 days (range: 848 days) for JT patients and 11 days (range: 2-50 days) for PT patients. The differences were not statistically significant (Kruskal-Wallis test, $p=0.989$ ). This was also the case when subgroup analysis was conducted according to the 


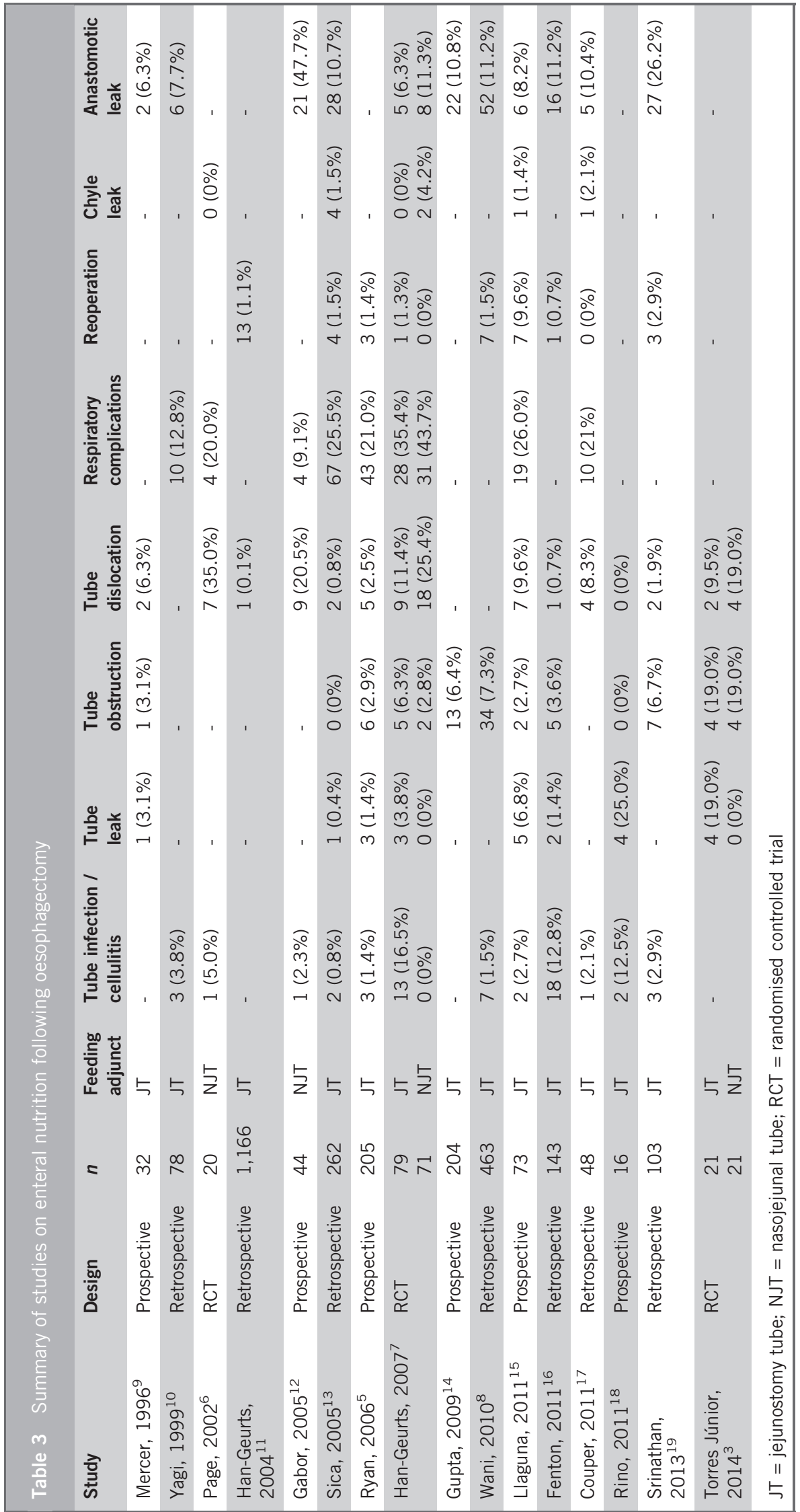


approach used for the abdominal part of the operation (Kruskal-Wallis test, $p=0.624$ and $p=0.429$ for open and laparoscopic respectively).

\section{Discussion}

Early EN following oesophagectomy is a key element in supporting a patient's recovery and reducing postoperative complications. Table 3 summarises studies on EN after oesophagectomy. ${ }^{3,5-19}$ Although the use of EN is not without its disadvantages, the benefits outweigh the risks.

Early EN was compared with delayed nutrition by Wang $e t$ $a l$, and was found to be associated with a short LOS, fewer incidences of pneumonia and better nutrition outcomes. ${ }^{20}$ The authors advocated starting enteral feeding within 48 hours of oesophagectomy. EN was compared with PN in a randomised controlled trial (RCT) by Takesue et al. ${ }^{21}$ They presented the data of 47 patients who had undergone a thoracic oesophagectomy. EN was found to have a positive impact on weight loss and decreased the incidence of pneumonia. A systematic review by Weijs et al including 17 studies on early oral feeding as well as NJT and JT feeding showed that early oral feeding was associated with a shorter LOS. ${ }^{2}$ NJT and JT patients had similar postoperative complication rates. However, nutritional outcomes and patient satisfaction were not reported.

Page et al compared NJT feeding following oesophagectomy with intravenous fluids. ${ }^{6}$ The incidence of NJT dislocation was $35 \%$ and cellulitis occurred in $20 \%$ of NJT patients. In the only RCT that investigated NJT versus JT feeding after oesophagectomy, Han-Geurts et al recruited 150 patients; 71 received NJT and 79 received JT feeding. ${ }^{7}$ The incidence of tube obstruction was $3 \%$ in the NJT group and $6 \%$ in the JT group. Tube dislocation was more prevalent among NJT patients (25\%) than among JT patients $(11 \%)$. There was no tube leak in the NJT group but three patients $(4 \%)$ developed tube leak in the JT group and one of these subsequently required a laparotomy. Cellulitis was not reported in the NJT group. Conversely, $16 \%$ of JT patients experienced cellulitis and wound infection.

In a RCT by Torres Júnior et al, 21 patients received NJT feeding while 21 received JT feeding. ${ }^{3}$ Both routes were associated with a similar number of complications and a similar LOS. Nevertheless, the JT route allowed EN for longer periods. The trial also included patients who underwent gastrectomy and pancreaticoduodenectomy. In a previous study from our unit on PT feeding after oesophagectomy, an incidence of $10 \%$ was reported for cellulitis and $1.5 \%$ for granuloma formation at the site of PT insertion. ${ }^{22}$ A retrospective review by Kent et al examining 38 patients who underwent PT insertion for different indications revealed that the most common associated complication was tube dislocation or migration $(24 \%){ }^{23}$ The authors concluded that PT feeding is a valid technique for the management of complications following oesophagectomy and that it was more tolerable than a NJT.

Our study demonstrated that early EN can be administered using three different routes with similar results. The
NJT route was the most commonly used technique, especially among laparoscopic cases $(82.9 \%)$. PT was the second most common technique for feeding and it was usually inserted during open oesophagectomy $(68.6 \%)$. JT was the least common route for feeding and it was mainly inserted using an open technique $(78.6 \%)$. The incidence of tube related complications in this study was $5.5 \%$ while that for 30-day morbidity was $58.9 \%$ (Table 2 ). The most prevalent tube related complication was cellulitis with an incidence of $3.3 \%$ across the entire cohort. NJT patients did not experience any tube related complications although the difference between the groups was not statistically significant (Table 2). The three different routes for EN did not appear to have an impact on LOS.

In our series, NJT and JT feeding did not lead to obstruction, dislocation or haemorrhage, and only one patient developed cellulitis in the JT group. PT feeding was associated with cellulitis, tube dislocation and haemorrhage. NJT obstruction was avoided in our cohort by four-hourly flushes of water through the feeding port. Tube dislocation may be prevented by stitching the NJT with a loose silk suture. If dislocation does occur, a new NJT can be inserted using an endoscope.

\section{Study limitations}

Our study has its own limitations as it was a retrospective analysis of a single centre practice. The group sizes were not balanced (fewer JT patients than NJT and PT patients) and the groups were heterogeneous in the types of surgery (laparoscopic vs open). Tube related complications occurred only in five patients, possibly owing to the sample size available. Finally, data on patient satisfaction with the different EN routes were not recorded during the hospital stay. A large prospective RCT is recommended to address these issues, and should focus on long-term nutritional outcomes and patients' experiences with the three EN routes following oesophagectomy.

\section{Conclusions}

NJT feeding is a feasible, effective technique that is not associated with major adverse events and it can be used safely for early EN following oesophagectomy. A multicentre prospective trial is advocated to verify these findings.

\section{References}

1. Online Analysis. International Agency for Research on Cancer. http://globocan. iarc.fr/Pages/online.aspx (cited June 2016)

2. Weijs TJ, Berkelmans GH, Nieuwenhuijzen GA et al. Routes for early enteral nutrition after esophagectomy. A systematic review. Clin Nutr 2015; 34: 1-6.

3. Torres Júnior LG, de Vasconcellos Santos FA, Correia MI. Randomized clinical trial: nasoenteric tube or jejunostomy as a route for nutrition after major upper gastrointestinal operations. World J Surg 2014; 38: 2,241-2,246.

4. Ryan AM, Rowley SP, Healy LA et al. Post-oesophagectomy early enteral nutrition via a needle catheter jejunostomy: 8-year experience at a specialist unit. Clin Nutr 2006; 25: 386-393.

5. Peng J, Cai J, Niu ZX, Chen LQ. Early enteral nutrition compared with parenteral nutrition for esophageal cancer patients after esophagectomy: a meta-analysis. Dis Esophagus 2016; 29: 333-341.

6. Page RD, Oo AY, Russell GN, Pennefather SH. Intravenous hydration versus naso-jejunal enteral feeding after esophagectomy: a randomised study. Eur $J$ Cardiothorac Surg 2002; 22: 666-672. 
7. Han-Geurts IJ, Hop WC, Verhoef C et al. Randomized clinical trial comparing feeding jejunostomy with nasoduodenal tube placement in patients undergoing oesophagectomy. Br J Surg 2007; 94: 31-35.

8. Wani ML, Ahangar AG, Lone GN et al. Feeding jejunostomy: does the benefit overweight the risk (a retrospective study from a single centre). Int J Surg 2010 8: 387-390.

9. Mercer CD, Mungara A. Enteral feeding in esophageal surgery. Nutrition 1996; 12: 200-201.

10. Yagi $\mathrm{M}$, Hashimoto $\mathrm{T}$, Nezuka $\mathrm{H}$ et al. Complications associated with enteral nutrition using catheter jejunostomy after esophagectomy. Surg Today 1999; 29: 214-218.

11. Han-Geurts IJ, Verhoef C, Tilanus HW. Relaparotomy following complications of feeding jejunostomy in esophageal surgery. Dig Surg 2004; 21: 192-196.

12. Gabor S, Renner H, Matzi V et al. Early enteral feeding compared with parenteral nutrition after oesophageal or oesophagogastric resection and reconstruction. Br J Nutr 2005; 93: 509-513.

13. Sica GS, Sujendran V, Wheeler J et al. Needle catheter jejunostomy at esophagectomy for cancer. J Surg Oncol 2005; 91: 276-279.

14. Gupta V. Benefits versus risks: a prospective audit. Feeding jejunostomy during esophagectomy. World J Surg 2009; 33: 1,432-1,438.

15. Llaguna $\mathrm{OH}, \mathrm{Kim} \mathrm{HJ}$, Deal $\mathrm{AM}$ et al. Utilization and morbidity associated with placement of a feeding jejunostomy at the time of gastroesophageal resection. $J$ Gastrointest Surg 2011; 15: 1,663-1,669.
16. Fenton JR, Bergeron EJ, Coello M et al. Feeding jejunostomy tubes placed during esophagectomy: are they necessary? Ann Thorac Surg 2011; 92 504-511.

17. Couper G. Jejunostomy after oesophagectomy: a review of evidence and current practice. Proc Nutr Soc 2011; 70: 316-320.

18. Rino $\mathrm{Y}$, Yukawa N, Murakami $\mathrm{H}$ et al. Primary placement technique of jejunostomy using the entristar ${ }^{\mathrm{TM}}$ skin-level gastrostomy tube in patients with esophageal cancer. BMC Gastroenterol 2011; 11: 8.

19. Srinathan SK, Hamin T, Walter $\mathrm{S}$ et al. Jejunostomy tube feeding in patients undergoing esophagectomy. Can J Surg 2013; 56: 409-414.

20. Wang G, Chen H, Liu J et al. A comparison of postoperative early enteral nutrition with delayed enteral nutrition in patients with esophageal cancer. Nutrients 2015; 7: 4,308-4,317.

21. Takesue $\mathrm{T}$, Takeuchi $\mathrm{H}$, Ogura $\mathrm{M}$ et al. A prospective randomized trial of enteral nutrition after thoracoscopic esophagectomy for esophageal cancer. Ann Surg Oncol 2015; 22(Suppl 3): S802-S809.

22. Katsoulis IE, Livingstone JI, Sagor GR. Tube pharyngostomy: a simple method for prolonged intubation of the foregut following oesophagogastric surgery. Dig Surg 2006; 23: 292-295.

23. Kent MS, Awais 0 , Schuchert MJ et al. Cervical pharyngostomy: an old technique revisited. Ann Surg 2008; 248: 199-204. 